\title{
ARTICLE
}

\section{The pangenome of an agronomically important crop plant Brassica oleracea}

\author{
Agnieszka A. Golicz ${ }^{1}$, Philipp E. Bayer ${ }^{2}$, Guy C. Barker ${ }^{3}$, Patrick P. Edger ${ }^{4}$, HyeRan Kim ${ }^{5}$, Paula A. Martinez ${ }^{1}$, \\ Chon Kit Kenneth Chan ${ }^{2}$, Anita Severn-Ellis ${ }^{2}$, W. Richard McCombie ${ }^{6}$, Isobel A.P. Parkin ${ }^{7}$, Andrew H. Paterson ${ }^{8}$, \\ J. Chris Pires ${ }^{9}$, Andrew G. Sharpe ${ }^{10}$, Haibao Tang ${ }^{11}$, Graham R. Teakle ${ }^{3}$, Christopher D. Town ${ }^{12}$, Jacqueline Batley ${ }^{2}$ \\ $\&$ David Edwards ${ }^{2}$
}

There is an increasing awareness that as a result of structural variation, a reference sequence representing a genome of a single individual is unable to capture all of the gene repertoire found in the species. A large number of genes affected by presence/absence and copy number variation suggest that it may contribute to phenotypic and agronomic trait diversity. Here we show by analysis of the Brassica oleracea pangenome that nearly $20 \%$ of genes are affected by presence/absence variation. Several genes displaying presence/absence variation are annotated with functions related to major agronomic traits, including disease resistance, flowering time, glucosinolate metabolism and vitamin biosynthesis.

\footnotetext{
${ }^{1}$ School of Agriculture and Food Sciences, The University of Queensland, Brisbane, Queensland 4072, Australia. ${ }^{2}$ School of Plant Biology, The University of Western Australia, 35 Stirling Highway, Crawley, Western Australia 6009, Australia. ${ }^{3}$ School of Life Sciences, The University of Warwick, Warwick CV35 9EF, UK. ${ }^{4}$ Department of Horticulture, Michigan State University, East Lansing, Michigan 48823, USA. ${ }^{5}$ Korea Research Institute of Bioscience \& Biotechnology, 125 Gwahak-ro, Yuseong-gu, Daejeon 34141, Korea. ${ }^{6}$ Cold Spring Harbor Laboratory, 1 Bungtown Road, Cold Spring Harbor, New York 11724, USA. ${ }^{7}$ Agriculture and Agri-Food Canada, 107 Science Place, Saskatoon, Saskatchewan, Canada S7N0X2. ${ }^{8}$ Plant Genome Mapping Laboratory, University of Georgia, Athens, Georgia 30602, USA. ${ }^{9}$ Division of Biological Sciences, Bond Life Sciences Center, University of Missouri, Columbia, Missouri 65211-7310, USA. ${ }^{10}$ National Research Council Canada, 110 Gymnasium Place, Saskatoon, Saskatchewan, Canada S7NOW9. ${ }^{11}$ Center for Genomics and Biotechnology, Fujian Provincial Key Laboratory of Haixia Applied Plant Systems Biology, Haixia Institute of Science and Technology (HIST), Fujian Agriculture and Forestry University, Fuzhou, Fujian 350002, China. ${ }^{12} \mathrm{~J}$. Craig Venter Institute, 9704 Medical Center Drive, Rockville, Maryland 20850, USA.

Correspondence and requests for materials should be addressed to D.E. (email: dave.edwards@uwa.edu.au).
} 
B rassica oleracea is a diploid, agronomically important plant species encompassing many popular crops, including cabbage, cauliflower, broccoli, Brussels sprout, kohlrabi and kale. Brassica crops display remarkable morphological diversity, and are grown for their inflorescences, axillary buds, leaves and stems. While two reference genomes of $B$. oleracea are available $^{1,2}$, a reference sequence cannot capture the entire gene content of a species owing to structural variants, namely the presence/absence variants (PAVs) and copy number variants ${ }^{3-6}$. Plant reference genomes have been shown to lack a number of agronomically important genes, restricting the gene space available for analysis, for example, in association studies 7 . To address this, pangenomes have been constructed for a number of species, including maize, rice and soybean ${ }^{7-9}$. The term pangenome was first introduced by Tettelin et al..$^{10}$ in 2005 and refers to a full genomic (genic) makeup of a species. Construction of a pangenome allows capturing of sequence affected by structural variation and possibly absent from the reference sequence of a single individual. A number of pangenome assembly approaches exist, including comparison of full de novo genome assemblies and reference guided assembly approaches ${ }^{6}$.

Here we describe the construction and analysis of a $B$. oleracea pangenome using nine morphologically diverse $B$. oleracea varieties and a wild relative-Brassica macrocarpa. The pangenome comprises 61,379 genes, $18.7 \%$ of which demonstrate PAV in the varieties analysed. Several of the variable genes are annotated with functions related to major agronomic traits, including disease resistance, flowering time, glucosinolate metabolism and vitamin biosynthesis, suggesting that PAVs may be important for the breeding of improved Brassica crops.

\section{Results}

Pangenome construction. The Brassica C pangenome was built using an iterative mapping and assembly approach, anchored by the publicly available genome of rapid cycling line TO1000 (ref. 2) and including additional sequences from nine other lines (eight cultivated lines and one wild type-B. macrocarpa, Supplementary Tables 1 and 2). The assembled pangenome is $587 \mathrm{Mbp}$ in size and contains 61,379 gene models, compared with the B. oleracea var TO1000 assembly of $488 \mathrm{Mbp}$ and 59,225 gene models (including 54,457 confident non-TE (transposable element) gene models used in the analysis; Supplementary Table 3 and Supplementary Fig. 1); and the $535 \mathrm{Mbp}$ assembly and 45,758 gene models reported for B. oleracea var capitata (cabbage) ${ }^{1,2}$. Among the contigs contributed by nine additional lines, $28 \%$ could be placed along the nine TO1000 chromosomes using paired read sequence information (Fig. 1 and Supplementary Fig. 2).

Gene presence/absence discovery and characterization. The majority $(81.3 \%, 49,895)$ of the pangenome is composed of core genes present in all lines, while $18.7 \%(11,484)$ of the genes are variable, with $2.2 \%(1,322)$ present in one line only (Supplementary Fig. 3). Modelling of pangenome size (Fig. 2) suggests a closed (restricted) pangenome with a finite number of genes (orthologous gene clusters), consistent with pangenome analyses in maize ${ }^{8}$ and soybean ${ }^{9}$. Variable genes were shorter than core genes, with fewer exons per gene (Fig. 3a,b and Supplementary Table 4), consistent with previous reports concerning genes displaying $\mathrm{PAV}^{11,12}$.

TE density surrounding core and variable genes was investigated. Higher TE density surrounding variable genes (compared with the core genes) was observed ( $U$-test, $P<0.001)$. In addition, a higher proportion of haT superfamily transposons and long interspersed nuclear elements in the vicinity of variable genes was observed (Supplementary Fig. 4). Long interspersed nuclear elements have previously been found to be associated with structural variants, and are thought to mediate structural variant generation via non-allelic homologous recombination ${ }^{13,14}$. Similarly, it was suggested that haT superfamily transposons mediate structural variant formation via alternative transposition ${ }^{15}$.

In total, 4,815,081 single-nucleotide polymorphisms (SNPs) were identified in the pangenome with an overall SNP density of 8.2 SNPs per $\mathrm{kb}$ (Supplementary Table 5). Private SNP abundance varied between B. macrocarpa and Cauliflower1 (Supplementary Fig. 5). There was greater SNP density within the coding regions of core genes than variable genes. However, when SNP density was adjusted for the number of instances of a gene, the variable genes had higher SNP density (Fig. 3c). Core genes have a greater proportion of synonymous SNPs and a lower proportion of nonsynonymous and nonsense SNPs than variable genes (Fig. 3d,e).

A phylogenetic tree of relationships between the 10 Brassica genotypes was built using RAxML (Fig. 4a). Overall, 4,324 (37.7\%) gene PAVs were consistent with the phylogenetic estimates of relationships and may represent morphotypelineage-specific gene PAV. The largest number of uniquely present and absent genes was found in B. macrocarpa, which reflects its greater evolutionary distance from the other samples ${ }^{16}$, while the line with the second greatest number of uniquely absent genes was the TO1000 rapid cycling line.

Functional analysis of variable genes. Functional analysis of variable genes suggests enrichment of genes and gene families involved in disease resistance, defence response, water homeostasis, amino-acid phosphorylation and signal transduction (Fig. 4b, and Supplementary Tables 6 and 7). PAV among defence response (biotic stress) genes has been observed in several plant species ${ }^{17,18}$. The presence/absence of resistance genes could partially be due to their overlapping roles and large number available for deletion following a whole-genome triplication event shared by the Brassica species ${ }^{1,19,20}$, however the presence of pathogens is also likely to impact gene retention due to strong selection for corresponding resistance genes. In total, 439 putative resistance genes were identified, including 251 core and 188 variable genes (Supplementary Fig. 6). The genes were classified in different categories based on presence of leucine-rich repeat (LRR), toll/interleukin-1 receptor-like (TIR) and coiled-coil (CC) domains (Supplementary Table 8). The genes were distributed unevenly across chromosomes, which is similar to observations made in other plants ${ }^{21,22}$, and an estimated $45 \%$ of nucleotide binding site (NBS) domain-containing genes were found in clusters.

Functional annotation of morphotype-lineage-specific PAV highlights genes involved in biotic and abiotic stress responses. These may reflect the evolution or breeding for adaptive traits. B. macrocarpa, which possesses a large number of uniquely present genes, has previously been identified as a potential donor of valuable traits ${ }^{23}$. Functional analysis suggests presence of unique genes involved in defence response, response to salt stress, cold and water deprivation (Supplementary Table 9).

Presence/absence variation of auxin-related genes. Wholegenome triplication contributed to expansion of gene families involved in auxin functioning ( $A U X, I A A, G H 3, P I N$, SAUR, TIR, $T P L$ and YUCCA), and morphology specification (TCP), and duplicated genes may contribute to the extraordinary morphological variation in Brassica species ${ }^{1}$. As PAV among those genes may also be a contributing factor, the homologues of auxinrelated genes and TCP were assessed. PAV within auxin-related genes but not TCP was detected (Supplementary Table 10). 


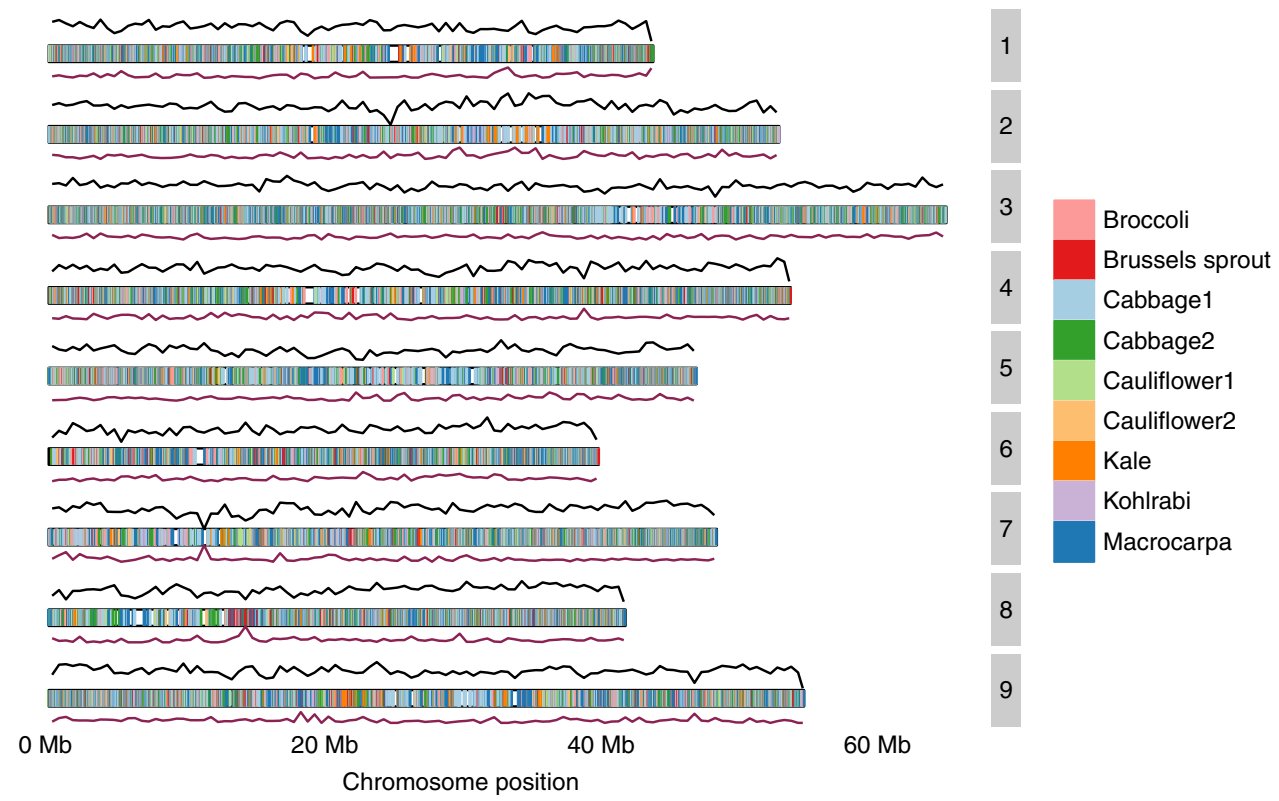

Figure 1 | B. oleracea pangenome. SNP density, pangenome contig placement on TO1000 chromosomes and variable gene density. Each chromosome is represented by three tracks, which from the top correspond to the following: (1) SNP density (black line) -each chromosome was split into 500 kb bins, the number of SNPs in bins is plotted as a function of bin position; (2) pangenome contig placement on TO1000 chromosomes (coloured rectangle)-contigs originating from each step of pangenome construction were placed along the chromosomes and colour coded according to the line; (3) variable gene density (burgundy line) - the number of variable genes in each $500 \mathrm{~kb}$ bin is divided by the total number of genes.
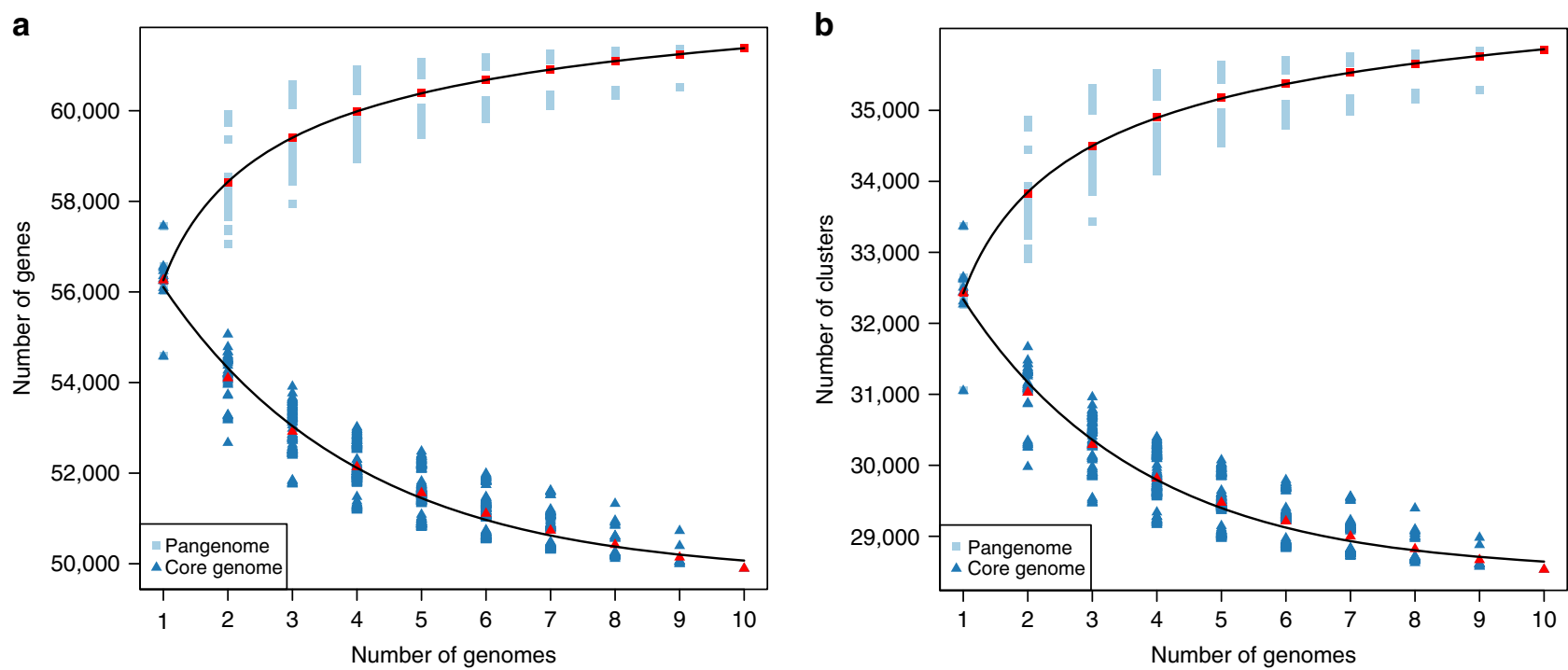

Figure 2 | Model describing the sizes of core and pangenome. Every genome added using (a) all genes and (b) orthologous gene clusters. Red points correspond to mean value. The pangenome size increases with each added line up to 61,379 genes (35,853 gene families), and extrapolation of pangenome size leads to a predicted pangenome of $63,865 \pm 31$ genes $(37,766 \pm 62$ gene families). The size of the core genome diminishes with every added line to 49,895 genes ( 28,532 gene families) with a predicted core genome size of $49,740 \pm 164$ genes ( $28,496 \pm 91$ gene families). \pm corresponds to s.e.

Presence/absence variation of flowering related genes. $B$. oleracea grows in a range of climatic zones and latitudes, and different cultivars have been selected for flowering time and maturity. There were 14 variable genes predicted to be involved in flowering, with TO1000 demonstrating the greatest absence of flowering genes (Supplementary Table 11 and Supplementary Fig. 7). A similar observation was made in Brassica rapa, where a rapid cycler was also missing several flowering time-related genes $^{24}$, suggesting that PAV may be a contributing factor of flowering time regulation. The genes identified include orthologues of genes encoding: MAF5, SEP2, ARP4, GID1B, FPF1-like, FHY1, GA2, GA3 and CO. Flowering locus $\mathrm{C}(F L C)$ is an important regulator of vernalization and flowering time. FLC is thought to control flowering in a dosage-dependent manner, and the flowering time variation appears to be affected by the number of copies of FLC gene present ${ }^{25}$. Only one FLC gene is present in Arabidopsis, whereas four, four and five genes have been identified in $B$. rapa, $B$. oleracea and $B$. napus, respectively ${ }^{26-28}$ A recent whole-genome assembly of $B$. napus allowed identification of nine FLC genes (four on the A genome and five on the C genome) and identified four FLC paralogues in B. oleracea var TO1000 (ref. 21). These four were identified in all lines examined, and two additional candidate FLC genes were discovered; one (BoFLC2) present in all lines except TO1000 and 
a

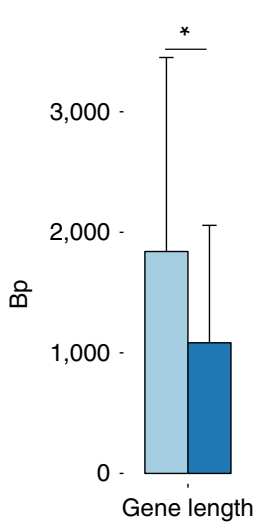

b

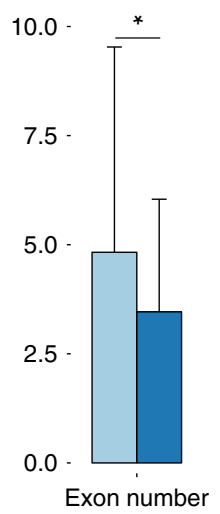

C

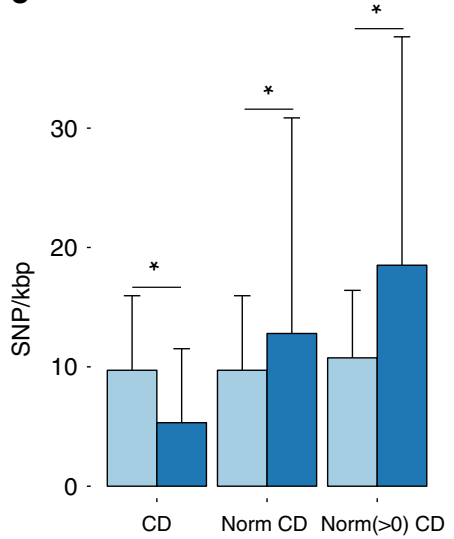

d

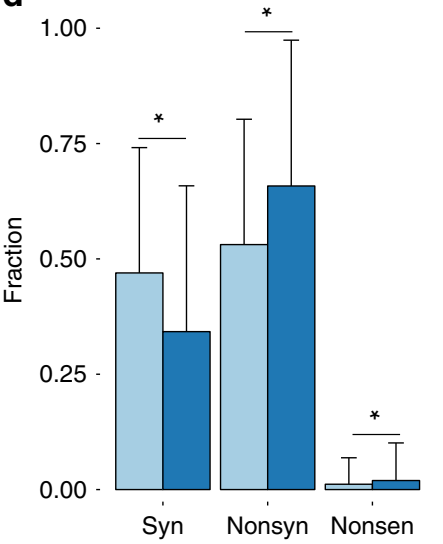

e

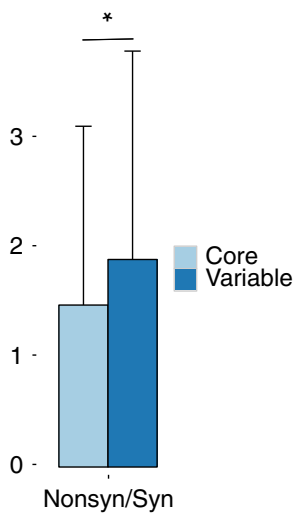

Figure 3 | Comparison of core and variable genomes. Core and variable gene properties, SNP density and difference between core and variable genes with respect to synonymous SNPs, nonsynonymous SNPs and nonsense SNPs. Variable genes are on average (a) shorter with (b) fewer exons and (c) lower SNP density. After correction for the number of instances of a gene, coding mean SNP density (Norm CD) of variable genes is higher than core genes, but the ranks of core genes are higher (U-test). When genes with at least one coding SNP are considered (Norm $(>0)$ CD), variable genes also have higher mean SNP density and the ranks of variable genes are higher (U-test). (d) There is a difference in the number of SNPs between core and variable genes within all three groups Syn (synonymous), Nonsyn (nonsynonymous) and Nonsen (nonsense). (e) Nonsyn/Syn ratio for core and variable genes. Bars correspond to means. Error bars correspond to s.d. ${ }^{\star} P<0.001$, U-test. The total number of genes considered $n=61,379$ (core, $n=49,895$; variable, $n=11,484$ ).

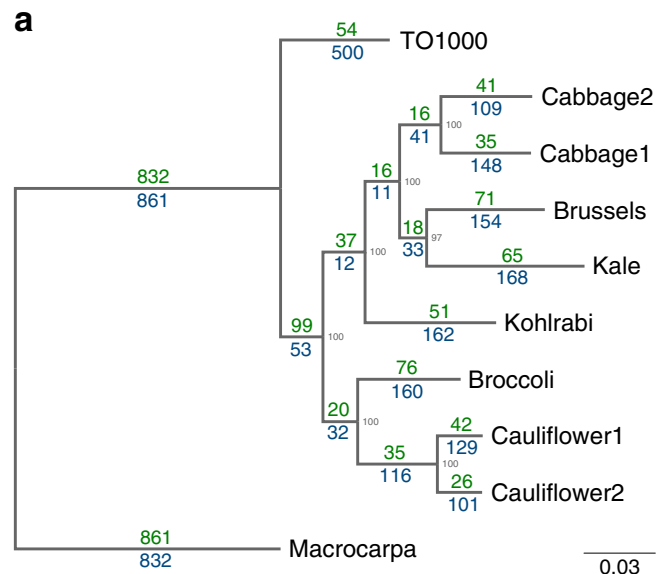

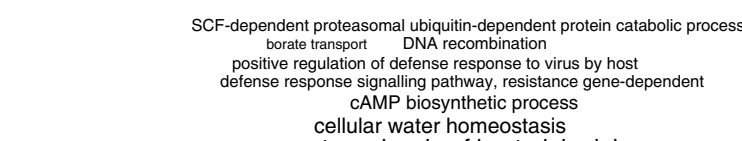
cellular water homeostasis response to molecule of bacterial origin defense response to bacterium, incompatible interaction cGMP biosynthetic process sesquiterpene biosynthetic process

defense response signalling pathway, resistance gene-independent response to nickel cation response to virus response to nickel cation response
transport of virus in host, tissue to tissue RNA 5'-end processing defense response to bacterium rotein dephosphorylation transmembrane receptor protein tyrosine kinase signalling pathway

Figure 4 | Phylogeny and gene ontology enrichment (a) Phylogenetic tree presenting relationships between the 10 varieties. The number of presence/ absence genes were placed on the phylogenetic tree, so that the branch leading to a node was labelled with the genes uniquely present and absent in all lines below the node. Genes absent are shown in blue and genes present in green. Scale bar indicates number of nucleotide substitutions per site.

(b) Significantly enriched gene ontology terms among variable genes using all pangenome genes as a background. Font size is proportional to -log( $p$ ).

the other (BoFLC5, partial gene model) present only in $B$. macrocarpa and Cauliflower1 (Fig. 5a). Studies in B. rapa have shown BrFLC2 to be a key regulator of flowering time ${ }^{29-31}$. In cauliflower, the disrupted BoFLC2 allele was associated with early flowering ${ }^{32}$. In addition, previous studies noted lack of hybridization of a BoFLC2 probe in a rapid cycling variety suggesting existence of an underlying deletion or substantial sequence variation ${ }^{27,28}$. The PAV analysis presented together with comparison of the contig harbouring the BoFLC2 gene with the TO1000 genome suggest that a deletion is a likely cause of BoFLC2 absence. Furthermore, it is a likely contributor to the early flowering phenotype of rapid cycler, TO1000.

Presence/absence variation of glucosinolate-related genes. The number of variable genes involved in biosynthesis and breakdown of secondary metabolites (glucosinolates, carotenoids, ascorbate, tocopherol and anthocyanin) was assessed (Supplementary
Tables 12 and 13, and Supplementary Fig. 8). In total, eight variable genes involved in glucosinolate biosynthesis and breakdown were observed. The variable genes included orthologues of genes involved in core structure formation, cytochrome CYP79A2, SUR1 and SOT18, and side chain modification (AOP2). Comparison of glucosinolates between Brassicas suggests significant variation in glucosinolate types and abundance ${ }^{33}$. Our analysis suggests that gene PAV may be a contributing factor to the diversity observed. Previous reports in Arabidopsis and other Brassica relatives have shown that gene duplications and subsequent sequence divergence contribute to glucosinolate pathway diversification ${ }^{21,34-36}$. AOP2 catalyses the conversion of methylsulphinylalkyl glucosinolates to corresponding alkenyl glucosinolates. It is of particular interest in Brassica crops because it catalyses conversion of health protective glucoraphanin, into deleterious products. In $B$. rapa, three functional, differentially expressed AOP2 genes were observed ${ }^{37}$. Broccoli harbours a non-functional AOP2 allele and accumulates glucoraphanin ${ }^{38}$. 

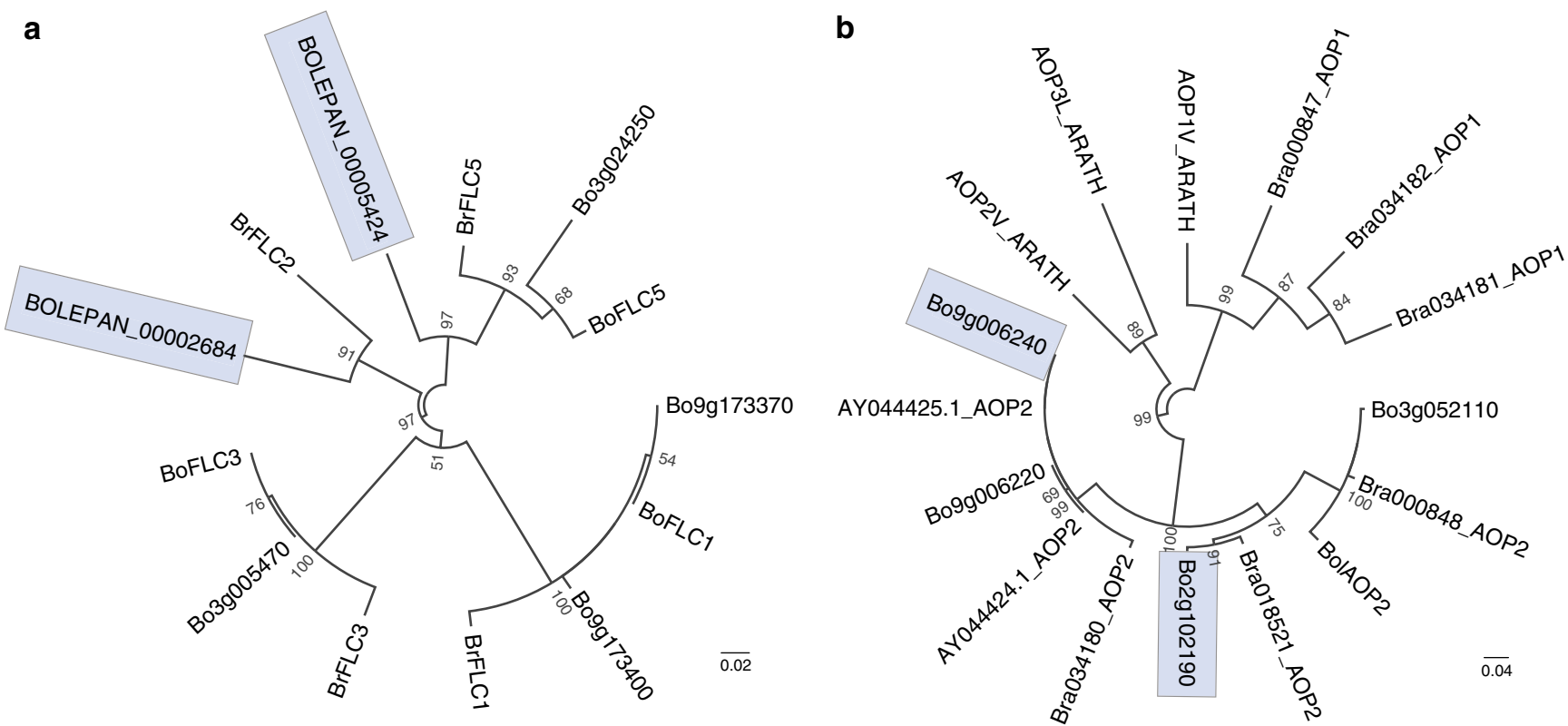

Figure 5 | Phylogenetic trees presenting relationships between $\mathbf{F L C}$ and AOP2 gene protein products. (a) There are six $F L C$ genes identified in the pangenome. BrFLC1, 2, 3, 5 and BoFLC1, 3, 5 are protein products of $B$. rapa and B. oleracea FLC genes, respectively, previously identified by Schranz et al. ${ }^{27}$. Bo3g005470, Bo3g024250, Bo9g173370 and Bo9g173400 are protein products of genes identified on the TO1000 portion, and BOLEPAN_00002684 and BOLEPAN_00005424 on the newly assembled portion of the pangenome. (b) There are four AOP2 genes identified in the pangenome. AOP1V_ARATH, AOP2V_ARATH and AOP3L_ARATH are protein products of $A O P 1,2,3$ genes identified in A. thaliana. BolAOP2 is a protein product of the only full-length $A O P 2$ gene identified in $B$. oleracea var capitata genome ${ }^{1}$. Proteins with IDs beginning with Bra are products of $A O P$ genes identified in $B$. rapa and proteins with IDs beginning with Bo are products of $A O P$ genes identified in the $B$. oleracea pangenome in this study. Genes Bo9g006220 and Bo9g006240 co-localize with quantitative trait locus (QTL) controlling amount of several glucosinolates ${ }^{70}$. Genes displaying PAV are highlighted in blue rectangles. Scale bars indicate number of amino-acid substitutions per site.

In cabbage, one full-length and two truncated $A O P 2$ proteins were reported ${ }^{1}$. In TO1000, four AOP2 genes were identified, two of these display PAV (absent in B. macrocarpa) (Fig. 5b). Two of the genes involved in ascorbate biosynthesis were variable (orthologues of L-galactose dehydrogenase), however none of the genes involved in carotenoid, tocopherol and anthocyanin biosynthesis were variable.

\section{Discussion}

The observation that nearly $18.7 \%$ of the pangenome is composed of variable genes may have implications for breeding. It is commonly recognized that different Brassica crop types have a restricted set of alleles compared with the wider species genepool, and here we show that some of these variations can be attributed to PAV. Performing wider crosses between crop types will give access to additional genes not present in a particular Brassica crop type. In addition, PAV may also contribute to the phenomenon of heterosis in F1 hybrids, since the presence of additional genes, even in heterozygous state, may give rise to increased vigour ${ }^{6,39,40}$. Finally, the finding that $B$. macrocarpa possesses the largest number of uniquely present genes suggests that Brassica wild relatives represent a significant source of new genes that were lost during domestication.

\section{Methods}

Pangenome assembly. Sequence data are listed in Supplementary Table 1. The pangenome was assembled using an iterative mapping and assembly approach. The approach is related to previously described reference-guided approaches ${ }^{7,8,41}$.

The iterative mapping and assembly strategy was chosen considering the nature of the data. Lack of long insert libraries resulted in highly fragmented whole-genome assemblies, which made whole-genome alignment challenging ${ }^{6}$. The publicly available reference sequence for a Chinese kale rapid cycling line (TO1000) ${ }^{2}$ was used as a reference for pangenome construction. The procedure involved three main steps: mapping of the reads to the reference sequence; assembly of the unmapped reads; and production of a new reference sequence by updating the old one with the newly assembled contigs. The mappings and assembly were performed in the following order: Cabbage1; Cabbage2; Kale; Brussels sprout; Kohlrabi; Cauliflower1; Cauliflower2; Broccoli; and Macrocarpa. Different orders were tested however the resulting assembly sizes were similar regardless of order used (Supplementary Fig. 9). Mapping was performed using Bowtie2 (ref. 42) v2.2.5 (--end-to-end --sensitive -I 0 -X 1000) and assembly was performed using MaSuRCA $^{43}$ v3.1.3. The TO1000 genome and the newly assembled contigs together constituted the pangenome. Mitochondrial (NC_016118.1) and chloroplast (NC_015139.1) genomes were included in the mappings (added to the TO1000 genome sequence) to eliminate potential plastid contamination. Before assembly, adapters were removed using Trimmomatic ${ }^{44}$ v0.36. The assembly was validated by remapping the reads to the assembly (Supplementary Figs 10-12)

Sequencing. For all samples, DNA was extracted from leaf tissue. For CA25, AC498, ARS_18 and HRIGRU011183, DNA was extracted using the megabasesized isolation protocol ${ }^{2}$. Illumina paired-end libraries with $300-500 \mathrm{bp}$ insert size were prepared following the manufacturer's instructions and sequenced using HiSeq2000 (100 and 101 bp reads) and HiSeq2500 (126 bp reads). For Badger Inbred 16, HRIGRU009617, BOL909 and B. macrocarpa, DNA was extracted using the QIAGEN DNeasy plant mini kit. Illumina paired-end libraries with 300-350 bp insert size were prepared following the manufacturer's instructions and sequenced using HiSeq2000. For Early Big, DNA was extracted using the CTAB procedure. Illumina paired-end library with $350 \mathrm{bp}$ insert size was prepared following the manufacturer's instructions and sequenced using the Genome Analyzer II.

Bacterial contamination. BLAST ${ }^{45}$ v2.2.30 against NCBI nt database (03.05.2016) was used to identify and remove potential contamination. Contigs whose best hits were not against green plants were tagged as contamination. Contaminant contigs were included in all the mappings but not included in the subsequent analysis.

Alignment of the newly assembled contigs to the pangenome. The newly assembled contigs from each stage of the assembly were aligned to the portion of the pangenome, which served as a reference at this stage using LASTZ v1.02.00 (--notransition --chain --ambiguous $=\mathrm{n}$--identity $=93$--coverage $=90$

--continuity $=95$ ). The sequence identity cutoff was estimated based on $B$. rapa and $B$. oleracea divergence. $B$. rapa and $B$. oleracea chromosomes 1 were aligned using LASTZ v1.02.00 (--chain --ambiguous $=\mathrm{n}$, genic regions were masked on both chromosomes). The per cent identity cutoff value was calculated as follows (estimated $B$. oleracea wild species divergence time) $\times($ first quartile $B$. rapa $-B$. 
oleracea per cent identity)/(estimated B. rapa - B. oleracea divergence time). The $B$. oleracea wild species divergence time used was $1.44 \mathrm{myr}$ ago (ref. 46). The B. rapa-B. oleracea divergence time used was $2.54 \mathrm{myr}$ ago (ref. 46 ).

Pangenome annotation. Newly assembled contigs $\geq 1,000 \mathrm{bp}$ in length were annotated using MAKER2 (ref. 47) v2.31.8. De novo gene prediction used SNAP48 and Augustus ${ }^{49}$, the EST evidence was based on B. oleracea genes downloaded from UniGene (ftp://ftp.ncbi.nlm.nih.gov/repository/UniGene/Brassica_oleracea/ Bol.seq.uniq.gz) and $95 \mathrm{k}$ ESTs (http://brassica.nbi.ac.uk/array_info.html), while protein evidence was based on Brassicaceae proteins downloaded from RefSeq. Publically available RNASeq data were downloaded from SRA and used as additional evidence. Sequence was masked against 'te_proteins.fasta' in the MAKER2 package. The total number of genes predicted may be underestimated due to lack of comprehensive RNASeq libraries for all the lines used in the analysis.

TE-related genes were detected using hmmsearch ${ }^{50} \mathrm{v} 3.1 \mathrm{~b} 2$ (trusted cutoff) using 137 identified TE-related domains ${ }^{51}$.

$\mathrm{R}$ genes were identified using hmmsearch v3.1b2 (trusted cutoff). Genes that contained PF00931 (NB-ARC) domain were considered to be R genes. The LRR and TIR domains were also assigned based on hmmsearch results. The CC domain was discovered using Paircoil2 (ref. 52). Resistance gene clusters were determined by their physical position order ${ }^{22,53,54}$. The parameter to define a cluster was two or more $R$ genes that occurred within a maximum of ten non- $R$ and $R$ genes. The neighbour joining tree was drawn using QuickTree ${ }^{55}$ v1.1.

Placement of contigs along T01000 chromosomes. The newly assembled contigs $>200$ bp in length were placed along the TO1000 chromosomes using paired-end read information. Reads from the nine varieties (Cabbage1, Cabbage2, Kale, Brussels sprout, Kohlrabi, Cauliflower1, Cauliflower2, Broccoli and Macrocarpa) were mapped to the pangenome (TO1000 genome and the newly assembled contigs) using Bowtie2 v2.2.5 (--end-to-end --sensitive -I 0 -X 1000). Duplicates were marked using Picard tools MarkDuplicates. Each of the mappings were processed separately looking only at contigs that originated from a given line. Reads mapping to the first and last $300 \mathrm{bp}$ of each newly assembled contig, which had mates mapping to a different chromosome/contig, were extracted using Samtools ${ }^{56}$. Only reads fulfilling the following criteria were extracted: MAPQ $\geq 10$ ( $-\mathrm{q} 10$ ); not mapped in proper pairs ( $-\mathrm{F} 2$ ); not unmapped ( $-\mathrm{F} 4$ ); mate not unmapped ( - F 8); and not duplicate ( - F 1024). All the extracted reads were inspected for the mapping position of the mate. If mates of $80 \%$ or more of the inspected reads from one end of a contig mapped to a single chromosome and the mapping positions of mates (leftmost mapping coordinate) did not span more than $1,000 \mathrm{bp}$, this end of the newly assembled contig was placed on the chromosome and assigned a position equal to the median of mates leftmost mapping coordinates. If both ends of a newly assembled contig were placed on a single chromosome, a lower placement coordinate was assigned. In case of conflicts no placement was made. Each placement had to be supported by at least 10 reads.

Gene presence/absence variation discovery. Gene presence/absence variation was characterized using the SGSGeneLoss package ${ }^{57}$. Reads from the 10 lines were mapped to the pangenome using Bowtie2 v2.2.5 (--end-to-end --sensitive -I 0 -X 1000). Reads from lines were subsampled to $\sim 25 \times$ using Seqtk v1.1. Only reads mapping in proper pairs were retained. SGSGeneLoss utilizes a depth-of-coverage calculation across all exons of the gene and calls gene absence when the horizontal coverage across exons (total number of exon bases covered by reads) of the gene was $<5 \%$. Only genes that were annotated on contigs with a length $\geq 1,000 \mathrm{bp}$ were used in this analysis. A gene was considered core if it was present in all lines and variable if it was absent in at least one line.

Presence/absence validation. Presence/absence gene calls were validated using PCR. Primers were designed for 28 genes (35 primers in total, Supplementary Tables 14 and 15). Presence/absence was tested in five varieties (Cabbage1, Brussels sprout, Cauliflower2, Kale and Kohlrabi).

\section{Gene clustering. Genes were clustered using OrthoMCL ${ }^{58}$ v2.09 (default} parameters). B. oleracea pangenome genes were clustered with $A$. thaliana genes ${ }^{59}$ (TAIR 10), and gene families were divided into core and variable. A gene family was considered to be core if at least one gene in the family was present in all the varieties. The gene family was considered variable if the whole gene family was missing from at least one line.

Pangenome modelling. Curves describing pangenome size, core genome size and new gene number for both individual genes and genes families were fitted in $\mathrm{R}$ using the nls function (nonlinear least squares) from package stats. Points used in regression corresponded to all the possible combinations of genomes. The combinations of genomes were obtained according to the following formula: $10 ! /(n !(10-n) !), n=[1,10]$, and the pangenome size was modelled using the power law regression $y=A x^{B}+C$ (refs 10,60). The core genome size was modelled using exponential regression $y=A e^{B x}+C$. The model was fitted using means.
TE annotation. TE elements were discovered using RepeatMasker ${ }^{61}$ using a $B$. oleracea repeat database. TE density surrounding genes was calculated as a proportion of base pairs annotated as TE in the 2,000 bp window preceding the start and following the end of gene.

SNP discovery. Mappings used for contig placement were also used for SNP discovery. Duplicates were marked using Picard tools MarkDuplicates. SNPs were discovered using Platypus ${ }^{62}$ v0.7.9.1 (--minMapQual $=30$--minBaseQual = 20). The SNP discovery model was diploid. The samples used were doubled haploids or highly inbred. Although a low percentage of SNPs are expected to be heterozygous, the vast majority of the SNPs are expected to be homozygous. Heterozygous SNPs were considered potential artefacts and removed. SNPs were categorized as coding synonymous, nonsynonymous and nonsense using R package VariantAnnotation ${ }^{63}$ v1.13.46.

Comparison of core and variable genes. Core and variable genes were compared with respect to gene length, exon number, coding SNP density, synonymous (not resulting in amino-acid change), nonsynonymous (resulting in amino-acid change), nonsense (introducing premature stop codon) SNP numbers and nonsynonymous/synonymous SNP ratio. All the pangenome genes were split into two groups corresponding to core and variable genes. The data did not meet parametric test assumptions and the groups were compared using Mann-Whitney $U$-test as implemented in R function wilcox.test (two-tailed test). No assumptions about similarity of shapes of distributions were made.

Phylogenetic analysis. Phylogenetic trees were constructed using RAxML ${ }^{64}$ v8.1.22 (-V -m ASC_GTRCAT --asc-corr = lewis -o Macrocarpa -p 12345 -\# 20). Bootstrapping was performed using 100 replicates.

Placement of genes on the phylogenetic tree. All the possible combinations of lines were analysed and for each combination lists of genes uniquely present and absent in these lines was obtained. For example combination BroccoliCauliflower1-Cauliflower2 will have two corresponding lists of genes: (1) genes found only in those three lines; the genes are present in all three lines, but absent in all the others; (2) genes absent only in those lines; the genes are absent in all three lines, but present in the others. The number of present and absent genes was then placed on the phylogenetic tree, so that the branch leading to a node was labelled with all the genes uniquely present and absent in all lines below the node.

Gene ontology annotation. The pangenome was functionally annotated using Blast2GO (ref. 65) command line v2.5. All the pangenome genes were compared with $A$. thaliana proteins pre-formatted to comply with Blast2GO naming requirements (ftp://ftp.arabidopsis.org/Sequences/blast_datasets/other_datasets/ CURRENT/At_GB_refseq_prot.gz). Comparisons were made using BLAST v2.2.30. Enrichment was performed using Fisher exact test as implemented in topGO ${ }^{66}$ package with method 'elim' used to adjust for multiple comparisons.

Detection of clusters enriched in variable genes. Clusters were constructed using OrthoMCL as described above. Clusters significantly enriched in variable genes were identified using Fisher exact test $(P$ value $<0.001)$. Functional annotation of clusters was performed by assigning functions of $A$. thaliana genes to the whole cluster.

Annotation of phylogeny-specific variable genes. Annotation of phylogenyspecific variable genes was done by counting the abundance of gene ontology terms for genes assigned to each node/branch.

Pathway annotation. The pathways involved in glucosinolate, carotenoid, ascorbate, tocopherol and anthocyanin biosynthesis and metabolism were identified from the A. thaliana metabolic pathway database (ftp://ftp.plantcyc.org/ Pathways/Data_dumps/PMN9_September2014/aracyc_pathways.20140902, version downloaded on 24.02.2015). The pathways and corresponding genes were extracted. Genes associated with flowering time listed in ref. 24 were downloaded. All the genes belonging to the pathways of interest and the flowering time genes were compared with the orthologous gene clusters. B. oleracea genes associated with pathways/processes were identified as follows: if a cluster contained an $A$. thaliana gene belonging to the pathway all the B. oleracea genes belonging to this cluster were extracted and assigned to the pathway. Subsequently, it was determined if the $B$. oleracea gene's best $A$. thaliana BLAST hit is directly involved in the pathway, if that was the case, the B. oleracea gene was deemed to be involved. The four B. oleracea FLC paralogues were taken from Chalhoub et al. ${ }^{21}$

Analysis of FLC and AOP genes. The B. rapa and B. oleracea FLC gene accessions were obtained from Schranz et al. ${ }^{27}$ The B. rapa and B. oleracea AOP genes were obtained from Liu et al. ${ }^{1}$ and Wang et al. ${ }^{67}$ A. thaliana $A O P$ proteins were obtained from Swiss-Prot. The sequences were aligned using Clustal Omega ${ }^{68}$ and 
a maximum likelihood tree with 500 bootstraps was constructed using MEGA6 (ref. 69).

Data availability. The code used for presence/absence detection have been made available at http://www.appliedbioinformatics.com.au/index.php/SGSGeneLoss. All sequencing data that support the findings of this study have been deposited in the National Center for Biotechnology Information Sequence Read Archive and are accessible through the SRA accession numbers PRJNA301390, PRJNA248388 and SRR074124. Additional data used in the study are available at http://www. appliedbioinformatics.com.au/index.php/BOLPANGENOME. All other data supporting the findings of this study are available in the article and its Supplementary Information files or are available from the corresponding author on request.

\section{References}

1. Liu, S. et al. The Brassica oleracea genome reveals the asymmetrical evolution of polyploid genomes. Nat. Commun. 5, 3930 (2014).

2. Parkin, I. et al. Transcriptome and methylome profiling reveals relics of genome dominance in the mesopolyploid Brassica oleracea. Genome Biol. 15, R77 (2014).

3. Morgante, M. et al. Gene duplication and exon shuffling by helitron-like transposons generate intraspecies diversity in maize. Nat. Genet. 37, 997-1002 (2005).

4. Gan, X. et al. Multiple reference genomes and transcriptomes for Arabidopsis thaliana. Nature 477, 419-423 (2011).

5. Cao, J. et al. Whole-genome sequencing of multiple Arabidopsis thaliana populations. Nat. Genet. 43, 956-963 (2011).

6. Golicz, A. A., Batley, J. \& Edwards, D. Towards plant pangenomics. Plant Biotechnol. J. 4, 1099-1105 (2016).

7. Yao, W. et al. Exploring the rice dispensable genome using a metagenome-like assembly strategy. Genome Biol. 16, 1-20 (2015).

8. Hirsch, C. N. et al. Insights into the maize pan-genome and pan-transcriptome. Plant Cell 26, 121-135 (2014)

9. Li, Y.-H. et al. De novo assembly of soybean wild relatives for pan-genome analysis of diversity and agronomic traits. Nat. Biotechnol. 32, 1045-1052 (2014).

10. Tettelin, H. et al. Genome analysis of multiple pathogenic isolates of Streptococcus agalactiae: implications for the microbial 'pan-genome'. Proc. Natl Acad. Sci. USA 102, 13950-13955 (2005).

11. Bush, S. J. et al. Presence/absence variation in A. thaliana is primarily associated with genomic signatures consistent with relaxed selective constraints. Mol. Biol. Evol. 31, 59-69 (2014).

12. Schatz, M. et al. Whole genome de novo assemblies of three divergent strains of rice, Oryza sativa, document novel gene space of aus and indica. Genome Biol. 15, 506 (2014).

13. Mills, R. E. et al. Mapping copy number variation by population-scale genome sequencing. Nature 470, 59-65 (2011).

14. Weckselblatt, B. \& Rudd, M. K. Human structural variation: mechanisms of chromosome rearrangements. Trends Genet. 31, 587-599 (2015).

15. Zhang, J., Zuo, T. \& Peterson, T. Generation of tandem direct duplications by reversed-ends transposition of maize Ac elements. PLoS Genet. 9, e1003691 (2013).

16. Song, K., Osborn, T. C. \& Williams, P. H. Brassica taxonomy based on nuclear restriction fragment length polymorphisms (RFLPs) : 3. Genome relationships in Brassica and related genera and the origin of B. oleracea and B. rapa (syn. campestns). Theor. Appl. Genet. 79, 497-506 (1990).

17. Xu, X. et al. Resequencing 50 accessions of cultivated and wild rice yields markers for identifying agronomically important genes. Nat. Biotechnol. 30, 105-111 (2012)

18. McHale, L. K. et al. Structural variants in the soybean genome localize to clusters of biotic stress-response genes. Plant Physiol. 159, 1295-1308 (2012).

19. Lysak, M. A. et al. Mechanisms of chromosome number reduction in Arabidopsis thaliana and related Brassicaceae species. Proc. Natl Acad. Sci. USA 103, 5224-5229 (2006).

20. Lysak, M. A., Koch, M. A., Pecinka, A. \& Schubert, I. Chromosome triplication found across the tribe Brassiceae. Genome Res. 15, 516-525 (2005).

21. Chalhoub, B. et al. Early allopolyploid evolution in the post-Neolithic Brassica napus oilseed genome. Science 345, 950-953 (2014)

22. Meyers, B. C., Kozik, A., Griego, A., Kuang, H. \& Michelmore, R. W. Genome-wide analysis of NBS-LRR-encoding genes in Arabidopsis. Plant Cell 15, 809-834 (2003).

23. Kole, C. Wild Crop Relatives: Genomic and Breeding Resources (Springer, 2011).

24. Lin, K. et al. Beyond genomic variation - comparison and functional annotation of three Brassica rapa genomes: a turnip, a rapid cycling and a Chinese cabbage. BMC Genomics 15, 250 (2014).

25. Osborn, T. C. The contribution of polyploidy to variation in Brassica species. Physiol. Plant. 121, 531-536 (2004).

26. Tadege, M. et al. Control of flowering time by FLC orthologues in Brassica napus. Plant J. 28, 545-553 (2001).

27. Schranz, M. E. et al. Characterization and effects of the replicated flowering time gene FLC in Brassica rapa. Genetics 162, 1457-1468 (2002).
28. Okazaki, K. et al. Mapping and characterization of FLC homologs and QTL analysis of flowering time in Brassica oleracea. Theor. Appl. Genet. 114, 595-608 (2007).

29. Zhao, J. et al. BrFLC2 (FLOWERING LOCUS C) as a candidate gene for a vernalization response QTL in Brassica rapa. J. Exp. Bot. 61, 1817-1825 (2010).

30. Kim, S.-Y et al. Delayed flowering time in Arabidopsis and Brassica rapa by the overexpression of FLOWERING LOCUS C (FLC) homologs isolated from Chinese cabbage (Brassica rapa L. ssp. pekinensis). Plant Cell Rep. 26, 327-336 (2007).

31. Xiao, D. et al. The Brassica rapa FLC homologue FLC2 is a key regulator of flowering time, identified through transcriptional co-expression networks. J. Exp. Bot. 64, 4503-4516 (2013).

32. Ridge, S., Brown, P. H., Hecht, V., Driessen, R. G. \& Weller, J. L. The role of BoFLC2 in cauliflower (Brassica oleracea var. botrytis L.) reproductive development. J. Exp. Bot. 66, 125-135 (2015).

33. Kushad, M. M. et al. Variation of glucosinolates in vegetable crops of Brassica oleracea. J. Agric. Food Chem. 47, 1541-1548 (1999).

34. Kliebenstein, D. J., Lambrix, V. M., Reichelt, M., Gershenzon, J. \& Mitchell-Olds, T. Gene duplication in the diversification of secondary metabolism: tandem 2-oxoglutarate-dependent dioxygenases control glucosinolate biosynthesis in Arabidopsis. Plant Cell 13, 681-693 (2001).

35. Hofberger, J. A., Lyons, E., Edger, P. P., Pires, J. C. \& Schranz, M. E. Whole genome and tandem duplicate retention facilitated glucosinolate pathway diversification in the mustard family. Genome Biol. Evol. 5, 2155-2173 (2013).

36. Edger, P. P. et al. The butterfly plant arms-race escalated by gene and genome duplications. Proc. Natl Acad. Sci. USA 112, 8362-8366 (2015).

37. Zhang, J. et al. Three genes encoding AOP2, a protein involved in aliphatic glucosinolate biosynthesis, are differentially expressed in Brassica rapa. J. Exp. Bot. 66, 6205-6218 (2015).

38. Li, G. \& Quiros, C. F. In planta side-chain glucosinolate modification in Arabidopsis by introduction of dioxygenase Brassica homolog BoGSLALK. Theor. Appl. Genet. 106, 1116-1121 (2003)

39. Springer, N. M. et al. Maize inbreds exhibit high levels of copy number variation $(\mathrm{CNV})$ and presence/absence variation $(\mathrm{PAV})$ in genome content. PLoS Genet. 5, e1000734 (2009).

40. Swanson-Wagner, R. A. et al. Pervasive gene content variation and copy number variation in maize and its undomesticated progenitor. Genome Res. 20, 1689-1699 (2010).

41. Schneeberger, K. et al. Reference-guided assembly of four diverse Arabidopsis thaliana genomes. Proc. Natl Acad. Sci. USA 108, 10249-10254 (2011).

42. Langmead, B. \& Salzberg, S. L. Fast gapped-read alignment with Bowtie 2. Nat. Methods 9, 357-359 (2012).

43. Zimin, A. V. et al. The MaSuRCA genome assembler. Bioinformatics 29, 2669-2677 (2013).

44. Bolger, A. M., Lohse, M. \& Usadel, B. Trimmomatic: a flexible trimmer for Illumina sequence data. Bioinformatics 30, 2114-2120 (2014).

45. Camacho, C. et al. BLAST $+:$ architecture and applications. BMC Bioinformatics 10, 421 (2009).

46. Arias, T., Beilstein, M. A., Tang, M., McKain, M. R. \& Pires, J. C. Diversification times among Brassica (Brassicaceae) crops suggest hybrid formation after 20 million years of divergence. Am. J. Bot. 101, 86-91 (2014).

47. Holt, C. \& Yandell, M. MAKER2: an annotation pipeline and genome-database management tool for second-generation genome projects. BMC Bioinformatics 12, 491 (2011)

48. Korf, I. Gene finding in novel genomes. BMC Bioinformatics 5, 1-9 (2004)

49. Stanke, M. et al. AUGUSTUS: ab initio prediction of alternative transcripts Nucleic Acids Res. 34, W435-W439 (2006).

50. Eddy, S. R. Accelerated profile HMM searches. PLoS Comput. Biol. 7, e1002195 (2011).

51. Piriyapongsa, J., Rutledge, M. T., Patel, S., Borodovsky, M. \& Jordan, I. K. Evaluating the protein coding potential of exonized transposable element sequences. Biol. Direct 2, 31-31 (2007).

52. McDonnell, A. V., Jiang, T., Keating, A. E. \& Berger, B. Paircoil2: improved prediction of coiled coils from sequence. Bioinformatics 22, 356-358 (2006).

53. Holub, E. B. The arms race is ancient history in Arabidopsis, the wildflower. Nat. Rev. Genet. 2, 516-527 (2001).

54. Richly, E., Kurth, J. \& Leister, D. Mode of amplification and reorganization of resistance genes during recent Arabidopsis thaliana evolution. Mol. Biol. Evol. 19, 76-84 (2002).

55. Howe, K., Bateman, A. \& Durbin, R. QuickTree: building huge NeighbourJoining trees of protein sequences. Bioinformatics 18, 1546-1547 (2002).

56. Li, H. et al. The sequence alignment/map format and SAMtools. Bioinformatics 25, 2078-2079 (2009).

57. Golicz, A. et al. Gene loss in the fungal canola pathogen Leptosphaeria maculans. Funct. Integr. Genomics 1-8 (2014).

58. Li, L., Stoeckert, C. J. \& Roos, D. S. OrthoMCL: Identification of ortholog groups for eukaryotic genomes. Genome Res. 13, 2178-2189 (2003) 
59. Initiative AG. Analysis of the genome sequence of the flowering plant Arabidopsis thaliana. Nature 408, 796-815 (2000).

60. Tettelin, H., Riley, D., Cattuto, C. \& Medini, D. Comparative genomics: the bacterial pan-genome. Curr. Opin. Microbiol. 11, 472-477 (2008).

61. Smit, A., Hubley, R. \& Green, P. RepeatMasker http://www.repeatmasker.org/ (2015).

62. Rimmer, A. et al. Integrating mapping-, assembly- and haplotype-based approaches for calling variants in clinical sequencing applications. Nat. Genet. 46, 912-918 (2014).

63. Obenchain, V. et al. VariantAnnotation: a Bioconductor package for exploration and annotation of genetic variants. Bioinformatics 30, 2076-2078 (2014).

64. Stamatakis, A. RAxML version 8: a tool for phylogenetic analysis and post-analysis of large phylogenies. Bioinformatics 30, 1312-1313 (2014).

65. Conesa, A. et al. Blast2GO: a universal tool for annotation, visualization and analysis in functional genomics research. Bioinformatics 21, 3674-3676 (2005).

66. Alexa, A., Rahnenführer, J. \& Lengauer, T. Improved scoring of functional groups from gene expression data by decorrelating GO graph structure. Bioinformatics 22, 1600-1607 (2006).

67. Wang, X. et al. The genome of the mesopolyploid crop species Brassica rapa. Nat. Genet. 43, 1035-1039 (2011).

68. ClustalOmega http://www.ebi.ac.uk/Tools/msa/clustalo/.

69. Tamura, K., Stecher, G., Peterson, D., Filipski, A. \& Kumar, S. MEGA6: molecular evolutionary genetics analysis version 6.0. Mol. Biol. Evol. 30, 2725-2729 (2013).

70. Sotelo, T., Soengas, P., Velasco, P., Rodríguez, V. M. \& Cartea, M. E. Identification of metabolic QTLs and candidate genes for glucosinolate synthesis in Brassica oleracea leaves, seeds and flower buds. PLoS ONE 9, e91428 (2014).

\section{Acknowledgements}

This work was supported by the Australian Research Council (Projects DP160104497, LP140100537, LP130100925, LP130100925 and LP110100200). Support is also acknowledged from the Australian Genome Research Facility (AGRF) and the Queensland Cyber Infrastructure Foundation (QCIF); the Pawsey Supercomputing Centre with funding from the Australian Government and the Government of Western Australia; resources from the National Computational Infrastructure (NCI), which is supported by the Australian Government and FlashLite supported by ARC LIEF grant LE140100061; The University of Queensland; Queensland University of Technology; Griffith University; Monash University; University of Technology Sydney; and The Queensland Cyber Infrastructure Foundation. J.C.P. acknowledges support from the USA National Science Foundation (NSF IOS 1339156). G.C.B. acknowledges DEFRA funding for the VeGIN project.

\section{Author contributions}

A.A.G., P.E.B. and P.A.M. designed analysis, performed analysis and wrote manuscript; G.C.B., P.P.E., H.R.K., W.R.M., I.A.P.P., A.H.P., J.C.P., A.G.S., H.T., G.R.T. and C.D.T. contributed data and wrote the manuscript; C.K.K.C. assisted with data analysis; A.S.-E performed PCR validation; D.E. and J.B. conceived research, designed analysis and wrote manuscript.

\section{Additional information}

Supplementary Information accompanies this paper at http://www.nature.com/ naturecommunications

Competing financial interests: W.R.M. has participated in Illumina-sponsored meetings over the past 4 years and has received travel reimbursement and an honorarium for presenting at these events. Illumina had no role in decisions relating to the study/work to be published, data collection, analysis of data and the decision to publish. W.R.M. has participated in Pacific Biosciences-sponsored meetings over the past 3 years and received travel reimbursement for presenting at these events. W.R.M. is a founder and shared holder of Orion Genomics, which focuses on plant genomics and cancer genetics. W.R.M. is an SAB member for RainDance Technologies, Inc. The remaining authors declare no competing financial interests.

Reprints and permission information is available online at http://npg.nature.com/ reprintsandpermissions/

How to cite this article: Golicz, A. A. et al. The pangenome of an agronomically important crop plant Brassica oleracea. Nat. Commun. 7, 13390 doi: 10.1038/ ncomms13390 (2016).

Publisher's note: Springer Nature remains neutral with regard to jurisdictional claims in published maps and institutional affiliations.

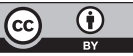

This work is licensed under a Creative Commons Attribution 4.0 International License. The images or other third party material in this article are included in the article's Creative Commons license, unless indicated otherwise in the credit line; if the material is not included under the Creative Commons license, users will need to obtain permission from the license holder to reproduce the material. To view a copy of this license, visit http://creativecommons.org/licenses/by/4.0/

(C) The Author(s) 2016 